\title{
Control of microcantilevers in dynamic force microscopy using time delayed feedback
}

\author{
$\operatorname{AUTHOR}(S):$
}

Yamasue, K; Hikihara, T

\section{CITATION:}

Yamasue, K ... [et al]. Control of microcantilevers in dynamic force microscopy using time delayed feedback. REVIEW OF SCIENTIFIC INSTRUMENTS 2006, 77(5): 053703.

\section{ISSUE DATE:}

2006-05

URL:

http://hdl.handle.net/2433/39815

\section{RIGHT:}

Copyright 2006 American Institute of Physics. This article may be downloaded for personal use only. Any other use requires prior permission of the author and the American Institute of Physics. 


\title{
Control of microcantilevers in dynamic force microscopy using time delayed feedback
}

\author{
Kohei Yamasue and Takashi Hikihara \\ Department of Electrical Engineering, Kyoto University, Katsura, Nishikyo, Kyoto 615-8510, Japan
}

(Received 5 February 2006; accepted 2 April 2006; published online 16 May 2006)

\begin{abstract}
It has been recently shown that microcantilever sensors in dynamic force microscopes possibly exhibit chaotic oscillations due to the nonlinear tip-sample interaction force. In this article, we propose elimination of the chaotic oscillations using the time delayed feedback control method, which has an ability to stabilize unstable periodic orbits embedded in chaotic attractors. An extended operating range of the microscopes is numerically estimated by stability analysis of the target periodic oscillation. We also discuss an improved transient response of oscillation, which allows us to accelerate the scanning rate of the microscopes without reducing their force sensitivity. () 2006
\end{abstract} American Institute of Physics. [DOI: 10.1063/1.2200747]

\section{INTRODUCTION}

The atomic force microscopy ${ }^{1}$ (AFM) has made remarkable advances toward a core technology for nanoscience and nanoengineering. $^{2}$ In particular, the dynamic force microscopy $^{3,4}$ (DFM) has been highly developed as a flagship operating mode of the AFM for this nearly two decades. ${ }^{5}$ In the DFM, a microcantilever vibrating at the resonance frequency is utilized as a force sensor to detect the interaction force between the tip manufactured at the free end and a sample surface facing the tip. ${ }^{3,4}$ The topography of the sample surface is imaged by raster scan of the surface with keeping the vibration or resonance frequency of the microcantilever constant. A broad range of samples has been observed so far in the resolution of atomic or molecular scale without damaging samples, including semiconducting, ${ }^{6-8}$ organic, ${ }^{9}$ and biological samples even in liquids. ${ }^{10,11}$ In addition, versatile applications of the vibrating microcantilever have been presented, such as profiling of surface properties, ${ }^{12-14}$ manipulation of single atoms and molecules, ${ }^{15}$ and control of surface structures. ${ }^{16}$

On the other hand, growing interests in the physical origin of high resolution imaging have shed light on nonlinear dynamics of microcantilever sensors vibrating near sample surface. ${ }^{17-19}$ In particular, much attention has been paid for microcantilevers in the DFM with amplitude modulation detection (AM-DFM) or tapping mode AFM. The tip of a microcantilever is exposed to a highly nonlinear force in an operating range of AM-DFM. ${ }^{20,21}$ As a result, a bistable behavior occurs in the proximity of sample surfaces. ${ }^{22}$ The involving jumping and hysteresis phenomena cause sudden and discontinuous transition of imaging characteristics. ${ }^{21}$ In addition, it was reported that the microcantilever exhibits subharmonic oscillation, period-doubling bifurcation, ${ }^{23,24}$ and chaotic oscillations. ${ }^{25-29}$ The resulting oscillation modes possibly reduce the force sensitivity of AM-DFM due to undesirable subharmonics and wide spread frequency spectrum, which are neglected in the standard device configuration of the AM-DFM. As for the chaotic oscillation, the operating range of the AM-DFM may be also limited by nonperiodic and irregular motion of the microcantilever. It is therefore significant to develop control techniques for microcantilever oscillations for improving the performance of AM-DFM. In this context, some motivated research groups have already proposed application of control techniques to microcantilever oscillation. ${ }^{25,26,30}$

In this article, we propose stabilization of the chaotic microcantilever oscillations using time delayed feedback control method. This control method was originally proposed by Pyragas in 1992 for stabilizing unstable periodic orbits embedded in chaotic attractors. ${ }^{31}$ Extensive studies on the control method have been carried out up to the present in the field of nonlinear dynamics. ${ }^{32}$ A key feature of the control method is that control input depends on only the difference signal between the current and past outputs of a nonlinear system. The control method, therefore, can be implemented without any identification of the model, system parameters, and underlying dynamics from experimental data. Here we numerically discuss application of the control method to the AM-DFM. It is firstly shown that the control method is able to extend operating range of the AM-DFM. The controlled AM-DFM is allowed to operate even in a parameter range, where chaotic microcantilever oscillations possibly occur without control. We secondly demonstrate that the control method has an ability to improve the transient response of microcantilever oscillation without reducing force sensitivity. Although transient behavior can be greatly influenced by the global structure of phase space especially in chaotic systems, ${ }^{33}$ nevertheless, the control input converging to null in steady state provides one way to overcome a trade-off between the scanning rate and force sensitivity of the AMDFM.

This article is organized as follows. Section II describes a mathematical model of vibrating microcantilevers near sample surfaces. A control input is additionally applied to the model of DFM originally proposed by Ashhab et al. ${ }^{26} \mathrm{Sec}-$ tion III presents the possibility of extending operating range of AM-DFM using control of microcantilever sensors. The 


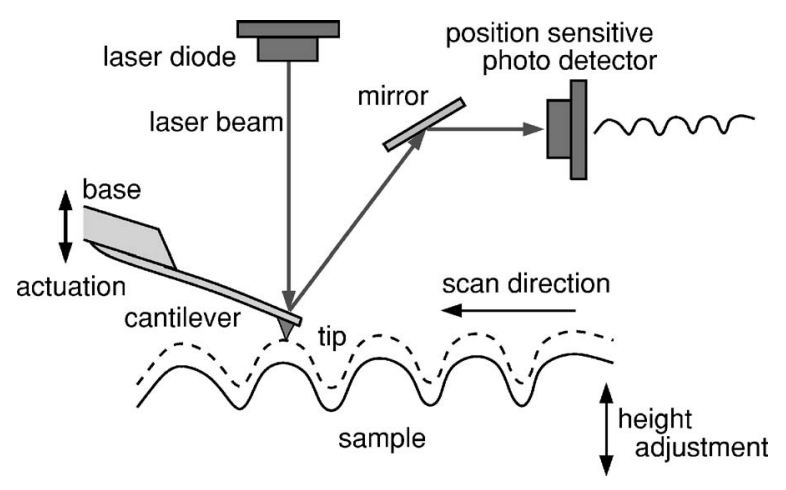

FIG. 1. Schematic diagram of dynamic force microscopy. Sample surface is scanned by microcantilever probe vibrating at its resonance frequency. During surface scan, mean distance between apex of tip and surface of sample is kept constant with a positioning device, which adjusts vertical position of surface, so that constant shift of resonance frequency is maintained. Time series of signal applied to positioning device provides topography of sample surface. Vibration of microcantilever is measured by optical lever method ${ }^{34}$ in standard device configuration.

stability of target periodic oscillation is numerically estimated based on the difference differential equation introduced in Sec. II. Using the same approach, Sec. IV discusses improvement of transient response of oscillating microcantilever sensors without reducing their force sensitivity.

\section{MODEL OF VIBRATING MICROCANTILEVER UNDER TIME DELAYED FEEDBACK CONTROL}

A schematic diagram of a DFM is shown in Fig. 1. A microcantilever vibrating at its resonance frequency is a force sensor in the DFM and detects the variation of tipsample interaction force as shift of the resonance frequency. Since the interaction force depends on the mean tip-sample distance, the shift of the resonance frequency estimates the variation of mean tip-sample distance. The topography of a sample surface is therefore traced by rasterscan of the surface with keeping the shift of resonance frequency constant. The constant shift of resonance frequency, or constant mean tip-sample distance, is achieved by adjusting the height of sample surface during the rasterscan with a positioning device, such as tube scanners. The time series of signal controlling the positioning device then gives a topography of the sample surface. The DFM has two major operating modes called AM-DFM ${ }^{3}$ and FM-DFM, ${ }^{4}$ in which the variation of amplitude and frequency is detected, respectively, to estimate the shift of resonance frequency. In both operating modes, variation of oscillation due to the shifted resonance frequency is measured using the optical lever method ${ }^{34}$ in the standard device configuration.

When the tip-sample interaction force is approximated by the Lennard-Jones potential, the first mode vibration of a microcantilever controlled by a scalar signal $u(t)$ is described by the following equation: ${ }^{26}$

$$
\begin{aligned}
\frac{d}{d t}\left[\begin{array}{l}
x \\
y
\end{array}\right] & \\
= & {\left[\begin{array}{c}
y \\
-x-\frac{d}{(\alpha+x)^{2}}+\frac{\Sigma^{6} d}{30(\alpha+x)^{8}}+\varepsilon(\Gamma \cos \Omega t-\Delta y)
\end{array}\right] } \\
& +\boldsymbol{b} u,
\end{aligned}
$$

where $x$ and $y$ denote the displacement and the velocity of tip, respectively. $\boldsymbol{b}$ denotes a two dimensional constant vector concerning coupling between the control input and the state variables. $\alpha$ is the equilibrium position of tip when the gravity only acts on it. $\Gamma$ and $\Omega$ correspond to the amplitude and frequency of the sinusoidal external force, which is provided to the microcantilever with the damping coefficient $\Delta$, respectively. $\Sigma$ denotes a constant related to the diameter of each molecule organizing the tip and the sample. It is noticed that Eq. (1) is dimensionless and $d=4 / 27$ is a constant derived in the course of eliminating the dimension. A small parameter $\varepsilon$ was prepared in Refs. 25 and 26 to prove the existence of a chaotic invariant set through the Melnikov method on Eq. (1) under $u(t)=0$. A chaotic oscillation of microcantilevers was subsequently presented numerically by Basso et al. based the same model. They showed a chaotic attractor arises following the cascade of period-doubling bifurcation. $^{28}$

As originally suggested by Pyragas, continuous control input $u(t)$ stabilizing a chaotic oscillation is given by the difference between the current output and the past one as follows: ${ }^{31}$

$$
u=K\left[g\left(x_{\tau}, y_{\tau}\right)-g(x, y)\right],
$$

where $\tau$ denotes the time delay and $K$ the feedback gain. The $g(x, y)=g[x(t), y(t)]$ and $g\left(x_{\tau}, y_{\tau}\right)=g[x(t-\tau), y(t-\tau)]$ imply a scalar output signal measured at the current time $t$ and the past time $t-\tau$, respectively. Since the control input (2) only depends on the output signal, the control method is easily implemented to experimental systems without any models of microcantilevers nor detailed analyses of underlying dynamics. So far, this control method has been successfully applied to various experimental systems including electronic circuits, ${ }^{35,36}$ laser systems, ${ }^{37}$ magnetoelastic beam, ${ }^{38}$ chemical systems, ${ }^{39}$ and gas-charge systems. ${ }^{40}$ In particular, the magnetoelastic beam ${ }^{41}$ and microcantilever under tip-sample interaction have a similar dynamical structure characterized by an elastic beam sinusoidally forced under two-well potential, although the dimension of the latter system is so much smaller. The time delay $\tau$ and feedback gain $K$ are important control parameters that substantially affect the control performance. The time delay $\tau$ is adjusted to the period of a target unstable periodic orbit we intend to stabilize in a chaotic attractor. The control input therefore converges to null after the controlled system is stabilized to the target orbit. The feedback gain governs the stability of the target orbit, although its systematic design has not been established. ${ }^{42}$

We hereafter investigate controlled dynamics of a microcantilever in the AM-DFM. The microcantilever has the damping coefficient $\Delta=0.4$ and is driven at fixed frequency $\Omega=1.0$, namely, its dimensionless mechanical resonance frequency. The remaining parameters are set as $\Sigma=0.3$ and $\varepsilon$ $=0.1$ based on the numerical result in Ref. 28. Assuming that the velocity of oscillation is measured as an output of the nonlinear system (1), the control signal $u(t)$ is given as follows: 


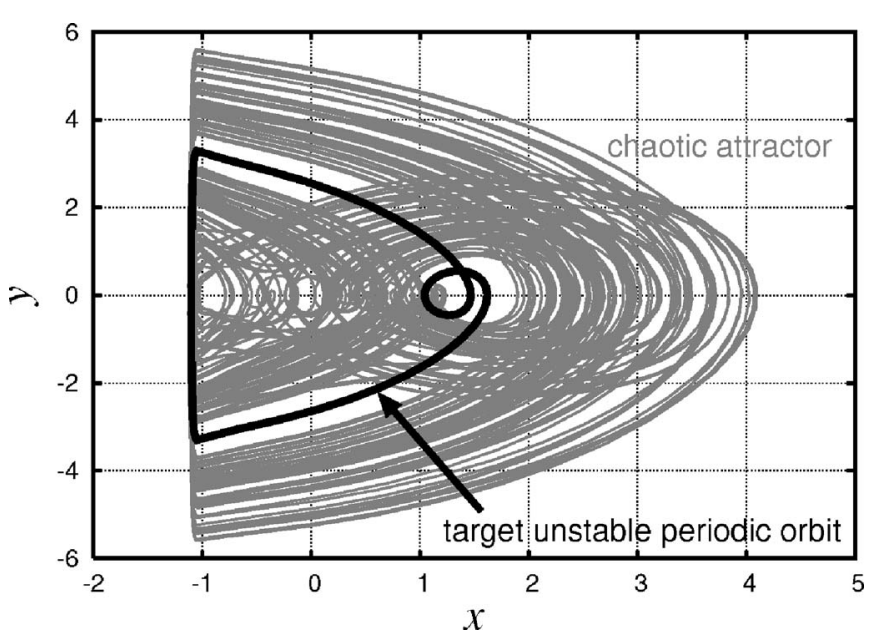

FIG. 2. Chaotic attractor reported by Basso et al. ${ }^{28}$ and target unstable periodic orbit embedded in it. The period of target orbit is $2 \pi$, which equals to the period of external force driving microcantilever sensor. The tip of microcantilever hits sample surface located $x=-\alpha=-1.2$ and then undergoes large repulsive force.

$$
u(t)=K[y(t-\tau)-y(t)] .
$$

This implementation of the control method is also obtained by putting $\boldsymbol{b}=\left[\begin{array}{ll}0 & 1\end{array}\right]^{T}, g(x, y)=y$ into Eqs. (1) and (2). The time delay $\tau$ is adjusted to $2 \pi / \Omega=2 \pi$ to stabilize an orbit with the same frequency as the external force oscillating the microcantilever. We note that the stabilization of this orbit is essential for the measurement by AM-DFM. This is because only this particular frequency component is detected in the standard device configuration using such as lock-in amplifiers and rms-dc converters with bandpass filters. A wide spread frequency spectrum due to the subharmonic and chaotic oscillation modes possibly decreases the force sensitivity of AM-DFM, as long as the frequency component corresponding to the driving frequency is detected. ${ }^{43}$ Besides, nonperiodic and irregular oscillation caused by chaos may also limit the resolution and operating range of the AMDFM.

\section{EXTENSION OF OPERATING RANGE}

Recently, experimental studies by Jamitzky et al. have demonstrated a chaotic oscillation of microcantilever in an actual AM-DFM, ${ }^{27,29}$ following the prediction by Ashhab et $a .^{25,26}$ and Basso et $a .^{28}$ In this section, we numerically show that the time delayed feedback control has a possibility to eliminate the chaotic oscillation from microcantilever sensors based on Eq. (1).

Figure 2 shows a chaotic attractor reported by Basso et al. for $\alpha=1.2$ and $\Gamma=20$ (Ref. 28) and an unstable periodic orbit embedded in it. The chaotic attractor and embedded unstable periodic orbit are shown by gray and black lines, respectively. This unstable periodic orbit has the same period as the driving signal and therefore should be stabilized for operation of the AM-DFM detecting the harmonic component of microcantilever for measurement, as mentioned Sec. II. The orbit is hereafter called target orbit. The target orbit is stabilized by adjusting the time delay $\tau$ to $2 \pi$ as shown in Fig. 3. The chaotic oscillation of tip is converted to the target

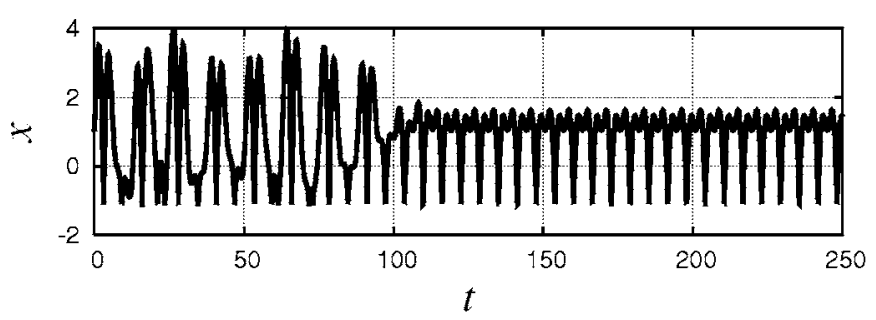

(a) Displacement of microcantilever

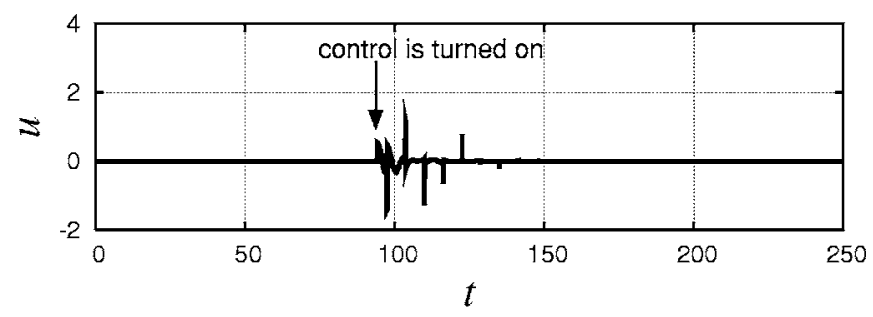

(b) Control input

FIG. 3. Stabilization of target unstable periodic orbit embedded in chaotic attractor using time delayed feedback control. (a) and (b) show temporal change of displacement of microcantilever and control signal, respectively. Control is activated at the time pointed by arrow in (b). The motion of microcantilever is irregular and nonperiodic before the activation. The control input finally converges to null after transient state, indicating that the activated control achieves convergence of motion to target periodic oscillation.

periodic one after the activation of control. The feedback gain is here adjusted to $K=0.2$ and time of activation is pointed by an arrow in Fig. 3(b). One can confirm that the displacement of tip shown in Fig. 3(a) is changed from chaotic to a periodic motion, as control input shown in Fig. 3(b) converges to null signal after the activation. Note that the null control signal after the activation implies the chaotic oscillation is eliminated by stabilizing the target unstable period- $2 \pi$ orbit embedded in the chaotic attractor. The control inputs, therefore, change nothing but the stability with respect to the target orbit. No system parameters are modified in contrast to the feedback control proposed in Refs. 25 and 26.

The time delayed feedback control is thus able to extend the operating range of AM-DFM. This is confirmed by Fig. 4 showing two different toned parameter ranges, which are numerically characterized based on the stability of the target orbit. The black area displays a parameter range, where the target period- $2 \pi$ orbit is unstable if control is not applied. It has been reported that this black area is not appropriate for operation of the AM-DFM due to the possibility of perioddoubling route to chaos. ${ }^{28}$ On the other hand, in the gray area, the control method can keep the target period- $2 \pi$ orbit stable. Note that this gray area completely includes the black one, although a part of the gray area is hidden behind the superimposed black area. We therefore conclude that the possibility of period-doubling bifurcation and subsequent chaotic oscillation is eliminated by stabilizing the target unstable periodic orbit. In other words, the operating range of AMDFM is extended by applying the control method to microcantilever. It should be mentioned that this extended operating range is limited by white areas outside the gray one. The boundary between two regions shows the saddle-node bifur- 


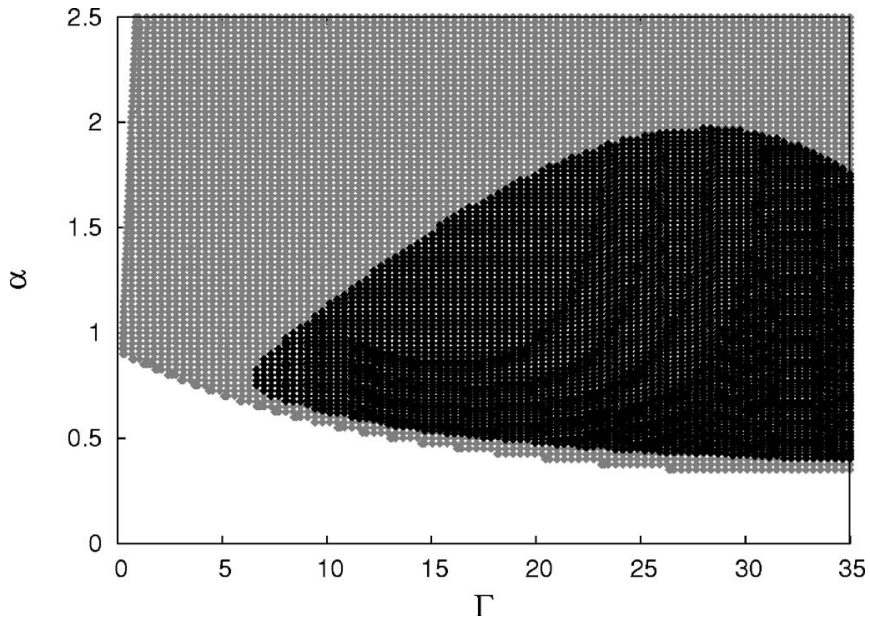

FIG. 4. Operating range of DFM under time delayed feedback control. Gray region shows parameter range where target orbit is kept stable under control. The region completely includes black area, in which operation of AM-DFM is not appropriate due to period-doubling route to chaotic oscillation reported in Ref. 28. Notice that a part of the gray area is hidden by superimposed black region. The boundary between gray and white regions for small $\alpha$ (short tip-sample separation) or tiny $\Gamma$ (small driving amplitude) shows saddle-node bifurcation curve of target orbit. Another stable periodic orbit can exist outside the gray region, but is not considered in this article.

cation curve of the target orbit. However, another period- $2 \pi$ orbit can be kept stable in the white region, although the target orbit to which we have referred does not exist due to its annihilation by the saddle-node bifurcation. It is also noted that the stability of the target orbit is here numerically determined by spectral radius of the target orbit. The spectral radius is the modulus of the characteristic multiplier that has maximal modulus among all characteristic multipliers. The spectral radius is estimated using the Newton-Picard method $^{44}$ and subspace shooting method ${ }^{45}$ for different differential equations. The target orbit is stable, if the spectral radius is less than unity.

In this section, we have numerically shown that time delayed feedback control can stabilize the unstable oscillation of microcantilevers. The stability analysis of target orbit has suggested the control method allows us to extend the operating range of AM-DFM. We should stress that control input changes only the characteristics multipliers of the target orbit and thereby oscillation in steady state are not modified regardless of control if the target orbit is stable in the uncontrolled system. This implies that time delayed feedback control has no influence on the force sensitivity and measured quantities of the AM-DFM. Based on this property, the next section discusses improvement of transient response of microcantilever oscillation using the control method.

\section{IMPROVEMENT OF TRANSIENT RESPONSE}

Slow scanning rate is a significant weakness of the DFM and thus various efforts have been made to overcome this weakness. ${ }^{46-48}$ As for the AM-DFM, the scanning rate is primarily limited by temporal length of the transient response of microcantilever and bandwidth of positioning device. ${ }^{47}$ In this section, we show the time delayed feedback control has an ability to improve the transient response of microcantile-

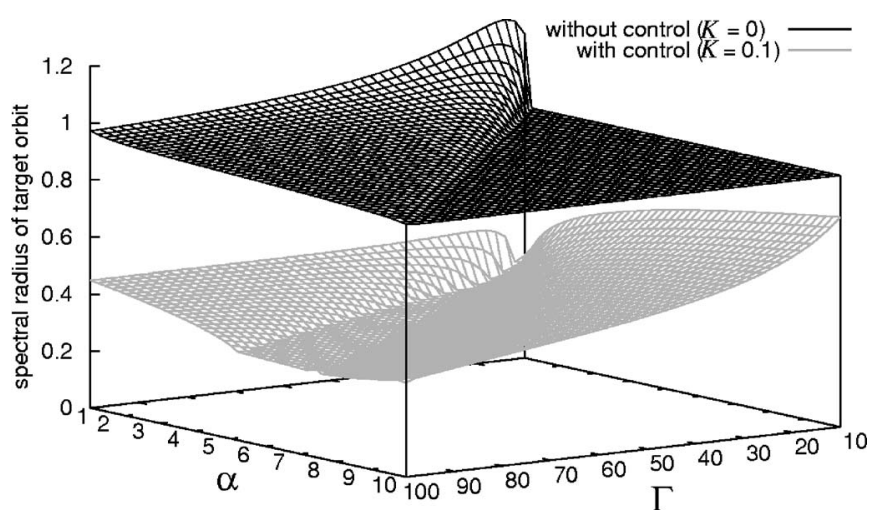

FIG. 5. Comparison between spectral radius of controlled (gray surface) and uncontrolled (black surface) target orbits. Spectral radius under control with $K=0.1$ is smaller than that under absence of control. Smaller spectral radius under control suggests that the time delayed feedback control reduces temporal length of transient response of microcantilever oscillation and then allows us to increase scanning rate of AM-DFM.

ver. The improved transient response allows us to accelerate the scanning rate of AM-DFM without reducing its force sensitivity.

The control input described by Eq. (3) explains the reason why the force sensitivity does not decrease under the time delayed feedback control. The reason is that the control input can effectively reduce the quality factor of microcantilever just in transient state toward the target periodic oscillation. Since the control input (3) includes a term $-K y(t)$ proportional to the velocity of tip, the control input serves as an apparent damping force applied to the microcantilever. On the other hand, the control input converges to null due to the effect of the term $K y(t-\tau)$ denoting the past velocity, as the oscillation converges to the target periodic oscillation. The control input has, therefore, no influence on the steady oscillation governing the force sensitivity of the microcantilever at all, while the apparent damping force improves the transient response, depending on the feedback gain. In other words, the time delayed feedback control allows us to overcome the trade-off between the transient response and force sensitivity. This is the essential difference from the active $Q$-controlled DFM, in which steady oscillation is changed by a feedback control. ${ }^{46,47}$

Figure 5 compares the spectral radius of target orbit in a parameter plane related to amplitude of excitation and displacement of sample surface. The spectral radius without control and under the control with $K=0.1$ is shown by a gray and black surfaces, respectively. The gray surface located below the black one illustrates that the transient response is improved under the time delayed feedback control. The spectral radius under control is smaller than that without control in the whole parameter region we here investigate. Since smaller spectral radius implies faster convergence to the target orbit, Fig. 5 evidently shows the transient response is improved by applying the time delayed feedback control.

We here estimate how much control contributes to the acceleration of scanning rate. The temporal length of transient response depends on the spectral radius and the initial variation of tip from the target orbit, if we assume the initial variation is sufficiently small such that the dynamics of mi- 
crocantilever is approximated by the linearized equation of Eq. (1). The motion of microcantilever close to the target oscillation is then described by the sum of dynamics in the direction of each invariant subspace. ${ }^{49,50}$ We additionally assume that the initial variation has only a component in the direction of the invariant subspace with respect to the characteristic multiplier that gives the spectral radius. The amplitude of variation decreasing in transient state is thereby estimated by the temporal change of a scalar variable:

$$
|\xi(t)|=|\rho|^{t / T}|\xi(0)|
$$

where $\xi(t)$ denotes the amplitude of variation and $\xi(0)$ is the initial variation from the target orbit. The $|\rho|$ is the spectral radius of the target orbit and $T=2 \pi / \Omega$ denotes the period of sinusoidal external force. Note that $\xi(t)$ shows attenuation in the direction of the particular invariant subspace. Although the temporal length of transient response is also related to the remaining invariant subspaces and even global structures of phase space, ${ }^{33}$ the estimation by Eq. (4) ensures the upper limit of the acceleration. The characteristic multiplier giving the spectral radius primarily determines the length of transient response. It is also noted that the variation itself is oscillatory if the $\rho$ has nonzero imaginary part. Nevertheless, the decay of its envelope is estimated by $\xi(t)$.

We define the settling time $T_{s}(K)$ as the elapsed time before the amplitude of variation reaches at $\varepsilon_{\mathrm{th}}|\xi(0)|$, where $\varepsilon_{\mathrm{th}}>0$ is small threshold and $K$ the feedback gain. The settling time is then obtained from Eq. (4) as follows:

$$
T_{s}(K)=T \frac{\log \varepsilon_{\mathrm{th}}}{\log |\rho(K)|} .
$$

We can thus estimate the ratio of the settling time without control to that with control. The ratio of the settling time is important because it gives the upper limit for acceleration of scanning rate under control input. Equation (5) implies that the ratio is given by

$$
\frac{T_{s}(0)}{T_{s}(K)}=\frac{\log |\rho(K)|}{\log |\rho(0)|},
$$

depending on neither the initial variation nor the threshold. Figure 6 shows the ratio of settling time numerically estimated for $K=0.1$. The parameter region here examined is the same as in Fig. 5. It is recognized that the ratio, continuously toned in Fig. 6, ranges from a few to 20. This implies the scanning rate can be a few to 20 times faster in the controlled case than in the uncontrolled one. The ratio, or upper limit, is significantly increased due to settling time $T_{s}(K)$ reduced by control input. It is noted that the ratio is here defined as positive infinity, because $T_{s}(0)=\infty$ if the uncontrolled target orbit is unstable. The trajectory without control does not converge to the unstable target orbit forever.

In this section, we have discussed improved transient response of the microcantilever under the time delayed feedback control. The resulting improved transient response enables us to accelerate scanning rate of the AM-DFM without reducing force sensitivity. The analysis on the spectral radius shows a few to 20 times faster scanning rate can be achieved using the time delayed feedback control.

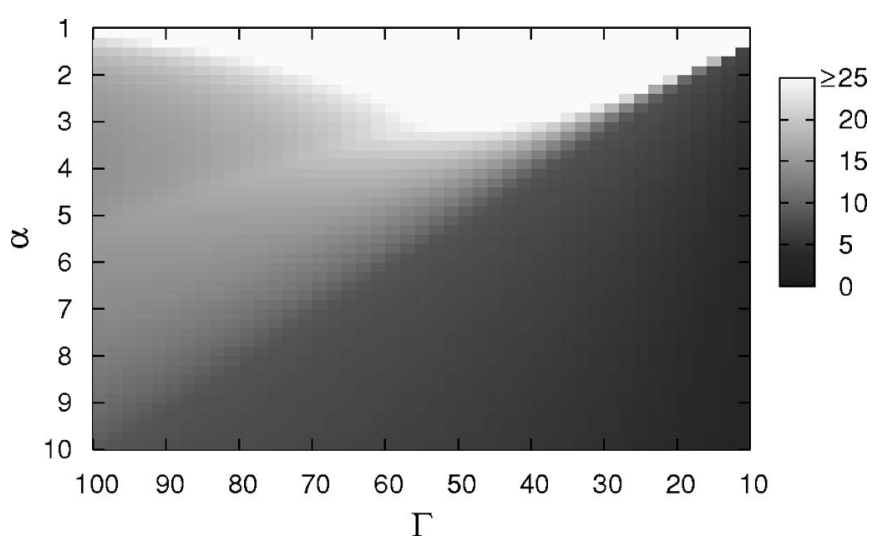

FIG. 6. Ratio of temporal length of transient response without control to that with control. The ratio (continuously toned in the figure) is numerically determined for $K=0.1$ based on Eq. (6) in the same parameter region as Fig. 5. The ratio estimates upper limit for acceleration of scanning rate under time delayed feedback control. A few to 20 times faster scanning rate can be achieved compared to uncontrolled case. This is due to reduced temporal length of transient response under control. Each axis has the same direction as that in Fig. 5.

\section{DISCUSSIONS}

In this article, we numerically discussed the stabilization of microcantilever probe in the DFM using time delayed feedback control. The operating range of DFM is extended by stabilizing the unstable periodic orbit embedded in the chaotic attractor. It implies that the intrinsic dynamics behind the instable motion including chaos can be utilized for the DFM measurement by applying the time delayed feedback control. Transient response in AM-DFM is also improved by the control method. We can therefore overcome the trade-off relationship between scanning rate and force sensitivity in the AM-DFM. It is pointed out that control eliminating the irregular oscillation can help manipulation of surface, since the manipulation seems to need strong tip-sample interaction that is obtained in the operation near sample surfaces. ${ }^{27,29} \mathrm{We}$ should note that this article focuses on stabilization of chaotic oscillation caused by homoclinic intersection and period-doubling bifurcation which has been predicted by Ashhab et al. ${ }^{25,26}$ and Basso et al., ${ }^{28}$ respectively. There is, however, another mechanism causing chaotic oscillation. Grazing bifurcation of tip oscillation in transition from noncontact to contact regime was predicted numerically by van de Water $e t a l .{ }^{51}$ and then suggested experimentally by Hu et $a l .{ }^{52}$ The time delayed feedback control could apply to a microcantilever grazing against the sample surface, although the possibility of application depends on dynamical properties of target periodic orbits under grazing bifurcation. The ongoing work is the implementation of the time delayed feedback control to an actual DFM.

\section{ACKNOWLEDGMENTS}

The authors would like to thank Professor Igor Mezić, University of California, Santa Barbara, for his kind acceptance for our use of DFM model. Our research is motivated by numerical results of his research group. The authors also thank Professor Kazumi Matsushige, Professor Hirofumi Yamada, and all other members of electronic material science 
and engineering laboratory, Kyoto University, for fruitful discussions and valuable suggestions on actual devices and their operation. The authors would like to appreciate Dr. Robert Walter Stark, Dr. Ferdinand Jamitzky, and Mr. Javier Rubio-Sierra, Ludwig-Maximilians-Universität München for their precious comments on nonlinear dynamics and control of microcantilevers in the DFM. This research is partially supported by the Ministry of Education, Culture, Sports, Science and Technology in Japan, The 21 st Century COE Program No. 14213201.

${ }^{1}$ G. Binning, C. F. Quate, and Ch. Gerber, Phys. Rev. Lett. 56, 930 (1986).

${ }^{2}$ F. J. Giessibl, Rev. Mod. Phys. 75, 949 (2003).

${ }^{3}$ Y. Martin, C. C. Williams, and H. K. Wickramasinghe, J. Appl. Phys. 61, 4723 (1987).

${ }^{4}$ T. R. Albrecht, P. Grütter, D. Horne, and D. Rugar, J. Appl. Phys. 69, 668 (1991).

${ }^{5}$ R. García and R. Pérez, Surf. Sci. Rep. 47, 197 (2002).

${ }^{6}$ Y. Sugawara, M. Ohta, H. Ueyama, and S. Morita, Science 270, 1646 (1995).

${ }^{7}$ S. Kitamura and M. Iwatsuki, Jpn. J. Appl. Phys., Part 2 34, L145 (1995).

${ }^{8}$ F. J. Giessibl, Science 267, 68 (1995).

${ }^{9}$ T. Fukuma, Ph.D. thesis, Kyoto University, 2003.

${ }^{10}$ P. K. Hansma et al., Appl. Phys. Lett. 64, 1738 (1994).

${ }^{11}$ C. A. J. Putman, K. O. Van der Werf, B. G. De Grooth, N. F. Van Hulst, and J. Greve, Appl. Phys. Lett. 64, 2454 (1994).

${ }^{12}$ Y. Martin and H. K. Wickramasinghe, Appl. Phys. Lett. 50, 1455 (1987).

${ }^{13}$ J. E. Stern, B. D. Terris, H. Mamin, and D. Rugar, Appl. Phys. Lett. 53, 2717 (1988)

${ }^{14}$ M. Nonnenmacher, M. P. O'Boyle, and H. K. Wickramasinghe, Appl. Phys. Lett. 58, 2921 (1991).

${ }^{15}$ Y. Sugawara, Y. Sano, N. Suehira, and S. Morita, Appl. Surf. Sci. 188, 285 (2002)

${ }^{16}$ N. Sasaki, S. Watanabe, and M. Tsukada, Phys. Rev. Lett. 88, 046106 (2002).

${ }^{17}$ N. Sasaki, M. Tsukada, R. Tamura, K. Abe, and N. Sato, Appl. Phys. A: Mater. Sci. Process. 66, S287 (1998).

${ }^{18}$ J. P. Aimé, R. Boisgard, L. Nony, and G. Couturier, Phys. Rev. Lett. 82, 3388 (1999)

${ }^{19}$ R. García and A. San Paulo, Phys. Rev. B 61, R13381 (2000).

${ }^{20}$ S. Rutzel, S. I. Lee, and A. Raman, Proc. R. Soc. London, Ser. A 459, 1925 (2003).

${ }^{21}$ R. García and A. San Paulo, Phys. Rev. B 61, R13381 (2000).

${ }^{22}$ P. Gleyzes, P. K. Kuo, and A. C. Boccara, Appl. Phys. Lett. 58, 2989 (1991).

${ }^{23}$ N. A. Burnham, A. J. Kulik, G. Germaud, and G. A. D. Briggs, Phys. Rev. Lett. 74, 5092 (1995).

${ }^{24}$ S. Patil and C. V. Dharmadhikari, Appl. Surf. Sci. 217, 7 (2003).

${ }^{25}$ M. Ashhab, M. V. Salapaka, M. Dahleh, and I. Mezić, Automatica 35,
1663 (1999)

${ }^{26}$ M. Ashhab, M. V. Salapaka, M. Dahleh, and I. Mezić, Nonlinear Dyn. 20, 197 (1999).

${ }^{27}$ F. Jamitzky, M. Stark, W. Bunk, W. M. Heckl, and R. W. Stark, in Proceedings of the Fourth IEEE Conference on Nanotechnology 2004 (IEEE, New York, 2004), pp. 38-40.

${ }^{28}$ M. Basso, L. Giarre, M. Dahleh, and I. Mezić, J. Dyn. Syst., Meas., Control 122, 240 (2000).

${ }^{29}$ F. Jamitzky, M. Stark, W. Bunk, W. M. Heckl, and R. W. Stark, Nanotechnology 17, S213 (2006).

${ }^{30}$ Y. Fang, D. Dawson, M. Feemster, and N. Jalili, Proceedings of 2002 ASME International Mechanical Engineering Congress and Exposition (ASME, New York, 2002), p. 33539.

${ }^{31}$ K. Pyragas, Phys. Lett. A 170, 421 (1992).

${ }^{32}$ W. Just, Handbook of Chaos Control (Wiley-VCH, New York, 1999), Chap. 2.

${ }^{33}$ C. Grebogi, E. Ott, and J. A. Yorke, Physica D 7, 181 (1983).

${ }^{34}$ G. Meyer and M. Amer, Appl. Phys. Lett. 53, 1045 (1988).

${ }^{35}$ J. E. S. Socolar, D. W. Sukow, and D. J. Gauthier, Phys. Rev. E 50, 3245 (1994).

${ }^{36}$ K. Pyragas and A. Tamaševičius, Phys. Lett. A 180, 99 (1993).

${ }^{37}$ S. Bielawski, D. Derozier, and P. Glorieux, Phys. Rev. E 49, R971 (1994).

${ }^{38}$ T. Hikihara and T. Kawagoshi, Phys. Lett. A 211, 29 (1996).

${ }^{39}$ P. Parmananda, R. Madrigal, M. Rivera, L. Nyikos, I. Z. Kiss, and V. Gáspár, Phys. Rev. E 59, 5266 (1999).

${ }^{40}$ T. Pierre, G. Bonhomme, and A. Atipo, Phys. Rev. Lett. 76, 2290 (1996).

${ }^{41}$ F. C. Moon and P. J. Holmes, J. Sound Vib. 65, 275 (1979).

${ }^{42}$ H. Nakajima, Phys. Lett. A 232, 207 (1997).

${ }^{43}$ Note that higher harmonics are available for imaging by AM-DFM. ${ }^{53}$ It has been also pointed out that detection of subharmonics has possibility to increase the force sensitivity of microcantilevers near period-doubling bifurcation $^{24,54}$ and period-adding bifurcation. ${ }^{51}$

${ }^{44}$ T. Luzyanina, K. Engelborghs, and K. Lust, Int. J. Bifurcation Chaos Appl. Sci. Eng. 7, 2547 (1997).

${ }^{45} \mathrm{H}$. Ito and A. Kumamoto, IEICE Trans. Fundamentals E81-A, 1791 (1998).

${ }^{46}$ J. Mertz, O. Marti, and J. Mlynek, Appl. Phys. Lett. 62, 2344 (1993).

${ }^{47}$ T. Sulchek et al., Appl. Phys. Lett. 76, 1473 (2000).

${ }^{48}$ N. Kodera, H. Yamashita, and T. Ando, Rev. Sci. Instrum. 76, 053708 (2005).

${ }^{49}$ S. Wiggins, Introduction to Applied Nonlinear Dynamical Systems and Chaos (Springer-Verlag, New York, 1990).

${ }^{50} \mathrm{~J}$. Guckenheimer and P. J. Holmes, Nonlinear Oscillations, Dynamical Systems, and Bifurcations of Vector Fields (Springer-Verlag, New York, 1983).

${ }^{51}$ W. van de Water and J. Molenaar, Nanotechnology 11, 192 (2000).

${ }^{52}$ S. Hu and A. Raman, Phys. Rev. Lett. 96, 036107 (2006).

${ }^{53}$ R. W. Stark and W. M. Heckl, Rev. Sci. Instrum. 74, 5111 (2003).

${ }^{54} \mathrm{~K}$. Yamasue and T. Hikihara, in Proceedings of 2005 International Symposium on Nonlinear Theory and its Applications (IEICE, Tokyo, 2005), pp. 582-585. 